Pacific Journal of Mathematics

RINGS WHOSE ADDITIVE SUBGROUPS ARE SUBRING 


\title{
RINGS WHOSE ADDITIVE SUBGROUPS ARE SUBRINGS
}

\author{
JOHN D. O’NEILL
}

In this paper the class (subclass) of associative rings whose additive subgroups are subrings (ideals) is completely characterized by defining relations. An exact description is also given of those rings in these classes which are commutative, regular, Artinian, Noetherian, or with identity. The only integral domains in either class are the ring of integers $Z$ and $Z /(p)$ for prime $p$.

0. Introduction. A ring which has the property, called $S$, that all its additive subgroups are subrings is called a $S$-ring. A ring $R$ is a $S$-ring if and only if, for every $x$ and $y$ in $R, x y$ is a linear combination of $x$ and $y$. This fact will be used constantly in this paper. All rings herein are associative, all groups are abelian, and all constants are integers. A ring $R$ will be primary, torsion, torsion-free, mixed, etc. exactly if its additive group, $R^{+}$, is primary, etc. By the rank of $R, r(R)$, we mean the rank of $R^{+}$. Occasionally, when the meaning is perfectly clear, distinction will not be made between $R$ and $R^{+}$. Other terminology is essentially that of [4]. Our chief results are found in Theorems $1.4,1.5,1.6,2.6,3.5$, and 3.6. Special $S$-rings are described in $\$ 4$.

1. Torsion rings. In this section all rings are torsion. We begin with primary rings.

Proposition 1.1. If $R$ is a p-primary $S$-ring, $R$ is bounded or $R$ is a zero-ring.

Proof. (a) Let $B$ be a basic subgroup of $R^{+}$and assume $B$ is unbounded. We prove $R^{2}=0$, Suppose $x^{2}=r x \neq 0$ for some $x$ in $B$ and $o(x)=p^{n}$. Choose $z$ independent of $x$ such that $o(z) \geqq p^{2 n}$. Then $\left(x+p^{n} z\right)^{2}=r x+p^{2 n} z=k\left(x+p^{n} z\right)$ for some $k$. This implies $p^{n}$ divides $k$ and $0=k x=r x$, a contradiction. Thus $x^{2}=0$, for every $x$ in $B$. Suppose $x$ and $y$ are linearly independent in $B$ and $x y=a x+b y$, by $\neq 0$. If $o(x)=p^{n}$ and $o(y)=p^{m}$, choose $z$ in $B$ such that $z$ is independent of $x$ and $y$ and $p^{n+m}$ divides $o(z)$. Write $x z=$ $c x+d z$. Then $x(y+z)=(a+c) x+(b y+d z)$. The right-hand term must be a multiple of $y+z$ which implies $d y=b y \neq 0$. But $p^{n} d z=$ $p^{n}(x z)=0$ and $p^{m}$ divides $d$ which implies $d y=0$. Therefore, $x y=0$ and $B^{2}=0$. It follows easily that $R^{2}=0$ (see Theorem 120.1 of 
[4]). (b) Assume, then, $B$ is bounded and write $R^{+}=D \oplus B$ where $D$ is divisible. Since $R$ is primary, $D^{2}=R D=D R=0$. It will suffice to show $B^{2}=0$ when $D \neq 0$. If $B$ is bounded by $p^{n}$, we select $z$ in $D$ such that $o(z) \geqq p^{2 n}$. Using this $z$ we repeat the proof given in (a) and again deduce $B^{2}=0$. This completes the proof.

If $R$ is a bounded primary ring, we wish to know when it has property $S$. The answer is given in the next proposition for which we need a lemma.

LEMMA 1.2. Let $R$ be a p-primary $S$-ring. If $x, y$ are linearly independent in $R, o(x) \leqq o(y), x^{2}=r x$, and $y^{2}=s y$, then $x y+y x=$ $s x+r y+e(x+y)$ for some constant $e$. If ex $\neq 0$, then $p=2,2 e x=0$, and $o(x)=o(y)$.

Proof. We write $(x+y)^{2}=k(x+y)$ and $(x+2 y)^{2}=1(x+2 y)$ where $k$ and 1 are constants. Solving simultaneously, we obtain $2 k \equiv$ $2(s+r)(\bmod o(x))$. If we set $k=s+r+e$, then $x y+y x=$ $s x+r y+e(x+y)$. If $e x \neq 0$, then $p=2$ and $2 e x=0$. In this event, $(x+2 y)^{2}=(r+2 s)(x+2 y)+2 e y$. Here 2ey must be a multiple of $x+2 y$ which implies $o(x)=o(y)$.

Proposition 1.3. Let $R$ be a ring on $G=\bigoplus\left\langle x_{i}\right\rangle$, a bounded $p$ group. Then $R$ has property $S$ iff there are constants $u_{i}$ and $v_{i}$ for each $i$ such that:

(1) $x_{i} x_{j}=v_{j} x_{i}+u_{i} x_{j}$, for every $i$ and $j$, or if $2^{n} G=0, r\left(2^{n-1} G\right)=2$ for some $n$,

(2) $x_{i} x_{j}=v_{j} x_{i}+u_{i} x_{j}$, for $i \neq j$ and $x_{i}^{2}=\left(u_{i}+v_{i}+2^{n-1}\right) x_{i}$, for every $i$.

Proof. (a) Sufficiency. Let $\alpha=\sum a_{i} x_{i}, \beta=\sum b_{j} x_{j}$ be elements in $R$. If (1) is given,

$$
\alpha \beta=\left(\sum_{j} b_{i} v_{j}\right) \alpha+\left(\sum_{i} a_{i} u_{i}\right) \beta
$$

and we are done. If (2) is given,

$$
\alpha \beta=\left(\sum_{j} b_{j} v_{j}\right) \alpha+\left(\sum_{i} a_{i} u_{i}\right) \beta+2^{n-1} \sum a_{i} b_{i} x_{i}
$$

The right-hand sum is a linear combination of $\alpha$ and $\beta$ if and only if $a_{i} b_{i} \equiv k a_{i}+1 b_{i}(\bmod 2)$ for some $k$ and 1 and all $i$. This, in turn, is possible if and only if $r\left(2^{n-1} G\right) \leqq 2$, as can be shown by considering cases. (b) Necessity. If $r(G)=1$, (1) is satisfied. We assume 
$r(G) \geqq 2$. We first show that, for each $i$, there exist $u_{1}^{\prime}$ and $v_{i}^{\prime}$ such that, for $i \neq j, x_{i} x_{j}=v_{j}^{\prime} x_{i}+u_{i}^{\prime} x_{j}$. For fixed $i$ choose $x_{k} \neq x_{i}$ of maximal order possible. Write $x_{i} x_{k}=c x_{i}+d x_{k}$ and, for $i \neq j \neq k, x_{i} x_{j}=a x_{i}+b x_{j}$. Then $x_{i}\left(x_{j}+x_{k}\right)=(a+c) x_{i}+\left(b x_{l}+d x_{k}\right)$. The right-hand term must be a multiple of $x_{j}+x_{k}$ and, hence, $d x_{j}=b x_{j}$. Similarly, if $x_{k} x_{i}=c_{0} x_{i}+d_{0} x_{k}$ and $x_{j} x_{i}=a_{0} x_{i}+b_{0} x_{j}$, an examination of $\left(x_{j}+x_{k}\right) x_{i}$ shows that $d_{0} x_{j}=$ $b_{0} x_{j}$. We let $u_{\imath}^{\prime}=d, v_{\imath}^{\prime}=d_{0}$ and do this for each $i$. Then, $x_{i} x_{j}=$ $v_{j}^{\prime} x_{i}+u_{i}^{\prime} x_{j}$ for $i \neq j$. Next, for each $i, x_{1}^{2}=\left(u_{i}^{\prime}+v_{i}^{\prime}+e_{i}\right) x_{i}$ for some $e_{i}$ such that $0 \leqq e_{1}<o\left(x_{i}\right)$. Write $r_{i}=u_{\imath}^{\prime}+v_{\imath}^{\prime}+e_{i \cdot}$. If $i \neq j$,

$$
x_{i} x_{j}+x_{j} x_{i}=\left(u_{j}^{\prime}+v_{\prime}^{\prime}\right) x_{i}+\left(u_{i}^{\prime}+v_{\imath}^{\prime}\right) x_{j}=r_{j} x_{i}+r_{i} x_{j}-\left(e_{j} x_{i}+e_{i} x_{j}\right) .
$$

If the right-hand term equals zero for all $i \neq j$, we let $u_{i}=u_{i}^{\prime}, v_{t}=v_{i}^{\prime}+e_{t}$ and condition 1 of the theorem will be satisfied. Suppose, for some $i \neq j$, $e_{j} x_{i}+e_{i} x_{j} \neq 0$ where $o\left(x_{i}\right) \leqq\left(x_{j}\right)$. If $e_{j} x_{i} \neq 0, o\left(x_{i}\right)=o\left(x_{j}\right)$ by (1.2). If $e_{i} x_{j} \neq 0, e_{l} x_{i}+e_{i} x_{l}$, as a multiple of $x_{i}+x_{j}$, equals $e_{i}\left(x_{i}+x_{l}\right)$. Here $e_{i} x_{i}=$ $e_{l} x_{i} \neq 0$, and again $o\left(x_{1}\right)=o\left(x_{l}\right)$. Since both terms in $e_{j} x_{t}+e_{i} x_{j}$ are nonzero, the sum would remain nonzero if, say, $i$ or $j$ were replaced by $k$ where $x_{k}$ has maximal order $p^{n}$ in $R^{+}$. It means $o\left(x_{i}\right)=o\left(x_{j}\right)=p^{n}$. By (1.2), $e_{1}$ and $e_{j}$ may be replaced by $2^{n-1}$ and $r\left(2^{n-1} G\right)=2$ (we already knew $\left.r\left(2^{n-1} G\right) \leqq 2\right)$. It follows that $x_{1}^{2}$ equals, for each $i,\left(u_{i}^{\prime}+v_{i}^{\prime}+2^{n-1}\right) x_{i}$. Let $u_{i}=u_{i}^{\prime}, v_{i}=v_{i}^{\prime}$ for every $i$. The proof is complete.

We now consider how a $S$-ring can be constructed on a bounded primary group. In general, if group $G=\bigoplus\left\langle x_{i}\right\rangle$, a ring (not necessarily associative) can be constructed on $G$ in the following way (see Theorem 120.1 of [4]). For each $i, j$, let $x_{i} x_{j}$ be an element in $G$ subject to the sole condition: $o\left(x_{i} x_{j}\right) \leqq \min \left(o\left(x_{i}\right), o\left(x_{j}\right)\right)$ and define multiplication on the rest of $G$ linearly. We note that these two conditions are also necessary. We may now state a theorem.

THEOREM 1.4. Let $G=\bigoplus\left\langle x_{i}\right\rangle$ be a bounded p-group where $o\left(x_{i}\right)=$ $n_{i}$. A $S$-ring can be constructed on $G$ by setting

(1) $x_{i} x_{j}=v_{j} x_{i}+u_{i} x_{j}$, and

(2) $\left(\sum a_{\imath} x_{\imath}\right)\left(\sum b_{j} x_{j}\right)=\sum_{\iota,} a_{i} b_{j} x_{i} x_{t}$, where

(3) $n_{i} u_{i} G=n_{i} v_{i} G=0$, and

(4) $u_{i} v_{j} x_{k}=0$ unless $i=j=k$.

If $p \neq 2$, any $p$-primary $S$-ring, not a zero-ring, is of this type.

Proof. By (1.3) and the preceding paragraph, conditions 1-3 are sufficient for constructing a $S$-ring (not necessarily associative) on $G$. Conversely, let $R$ be a $p$-primary $S$-ring, not a zero-ring. Its additive group has the form $G$ by (1.1) and, by (1.2) and the paragraph above, conditions 1-3 are necessary. We now show that (4) is necessary 
and sufficient for associativity. Multiplication is associative if and only if, for all $i, j, k,\left(x_{i} x_{j}\right) x_{k}=x_{i}\left(x_{j} x_{k}\right)$ or, by computation, if and only if $u_{j} v_{k} x_{i}=v_{j} u_{i} x_{k}$ for every $i, j, k$. Clearly, (4) implies this equation. Conversely, suppose this equation is given. If $i \neq k, x_{i}$ and $x_{k}$ are linearly independent and $u_{i} v_{j} x_{k}=0$. If $j \neq k$, we substitute, in the above equation, $k, i, j$ for $i, j, k$ respectively, and derive $u_{i} v_{j} x_{k}=v_{i} u_{k} x_{j}$, and again $u_{i} v_{j} x_{k}=0$. Property (4) is established.

REMARK. A $S$-ring on a 2-group, however, need not satisfy condition 1 of the preceding theorem. An example is the ring-direct sum $Z /(2) \oplus Z /(2)$, which we call $M$. The following theorem will allow for the ring $M$.

THEOREM 1.5. Let $G$ be as in (1.4) where $2^{n} G=0$ and $r\left(2^{n-1} G\right)=2$ for some $n$. A $S$-ring $R$ can be constructed on $G$ by setting

(1') $x_{i} x_{j}=v_{j} x_{i}+u_{1} x_{j}$, for $i \neq j$ where $x_{i}^{2}=\left(u_{1}+v_{i}+2^{n-1}\right) x_{i}$, for every $i$,

(5) all $u_{\imath}, v_{\imath}$ are even,

and satisfying conditions 2-4 of (1.4). A S-ring on a 2-group, not a zero-ring, has this construction or that of (1.4).

Proof. (a) Let $G$ and multiplication on $G$ be as given. Conditions $1^{\prime}, 2$, and 3 define a $S$-ring $R$ (not necessarily associative) on $G$ for the same reasons as in (1.4). We claim (4) and (5) ensure $x_{i}\left(x_{j} x_{k}\right)=\left(x_{i} x_{j}\right) x_{k}$ for all $i, j, k$. If $i=j=k$, this is clear. If $i, j, k$ are all distinct, these products are computed exactly as in (1.4) and the equation is true by (4). Suppose, in the equation, exactly two subscripts agree. Again, the value of each product in $G$ is as in (1.4). This is so because each term involving the number $2^{n-1}$ disappears. We illustrate this with one example. In $x_{i}\left(x_{i} x_{j}\right)=v_{j}\left(u_{i}+v_{i}+2^{n-1}\right) x_{i}+u_{i} x_{i} x_{j}, \quad v_{j} 2^{n-1} x_{i}=0 \quad$ by (5). Therefore, $R$ is associative. (b) We now verify the last sentence of the theorem. Suppose $R$ is a $S$-ring on a 2 -group $G$ but is neither a zero-ring nor structured as in (1.4). By (1.3), (1') is necessary together with (2) and (3). If all $u_{i}$ and $v_{i}$ are even, the products $x_{i}\left(x_{j} x_{k}\right)$ and $\left(x_{i} x_{j}\right) x_{k}$ are computed as in (1.4) unless $i=j=k$ and (4) is again needed. If (5) is not satisfied, we will prove $R$ is isomorphic to $M$, the ring mentioned in the Remark above. That $M$ has a construction satisfying the conditions of this theorem is routine to show. Therefore, suppose (5) is not satisfied and $u_{i}$ or $v_{i}$ is odd for fixed $i$. Consider $x_{i}^{2} x_{j}=x_{i}\left(x_{i} x_{j}\right)$ for $j \neq i$ and $o\left(x_{l}\right)=p^{n}$. If we compute this equation in $G$ and equate the two $x_{j}$-terms, we obtain $\left(u_{i}+v_{t}+2^{n-1}\right) u_{i} x_{j}=u_{i}^{2} x_{j}$ or $\left(v_{i}+2^{n-1}\right) u_{i} x_{j}=0$. Similarly, from $x_{j} x_{i}^{2}=\left(x_{j} x_{i}\right) x_{i}$, we obtain $\left(u_{i}+2^{n-1}\right) v_{i} x_{j}=0$. We deduce from these equations that $u_{i}$ and $v_{i}$ have the same parity. Thus, $u_{i}$ and $v_{i}$ are both odd, $\left(v_{i}+2^{n-1}\right) x_{j}=0$, and $n=1$. Also, $x_{i}^{2}=x_{i}$ and $x_{j}^{2}=x_{j}$. From the condition on rank, it follows 
that $R^{+}=\left\langle x_{i}\right\rangle \oplus\left\langle x_{j}\right\rangle$. We claim $x_{i} x_{j}=x_{j}=x_{j} x_{i}$. Suppose $x_{i} x_{j}=x_{i}+x_{j}$ (the only other possibility since $u_{i}$ is odd). Then $x_{i}+x_{j}=x_{1}^{2} x_{j}=$ $x_{i}\left(x_{i} x_{J}\right)=x_{j}$, a contradiction. Thus, $x_{i} x_{j}=x_{j}$. Similarly, $x_{j} x_{i}=x_{l}$. By writing $R^{+}=\left\langle x_{i}+x_{j}\right\rangle \oplus\left\langle x_{j}\right\rangle$, we readily see that $R$ is isomorphic to $M$ which satisfies the conditions of the theorem.

Remark. At first glance, the construction of $S$-rings might seem unduly complicated, but this is not so. Suppose $G$ is the group given in (1.4) with least bound $p^{N}$. We can satisfy the conditions of the theorem by, for each $i$, letting $u_{t}=r_{i}, v_{\imath}=0$ where $p^{N}$ divides $r_{t} n_{l}$. Another example shows that all $u_{i}, v_{i}$ can be nonzero. Let $R$ be the ring on the group $\langle x\rangle \oplus\langle y\rangle$ where $o(x)=81=o(y), \quad x^{2}=27 x, \quad y^{2}=27 y, \quad x y=$ $9 x+18 y$, and $y x=18 x+9 y$. This ring also has property $S$.

We now consider torsion $S$-rings in general.

THEOREM 1.6. Let $G$ be a torsion group with primary decomposition, $G=\bigoplus_{p} G_{p} . \quad A$ ring $R$ on $G$ has property $S$ if and only if, for each $p$, the subring $R_{p}$ on $G_{p}$ has property $S$.

Proof. The necessity of the condition is clear. We suppose $R_{p}$ is a $S$-ring for each prime $p$ and prove $R=\oplus R_{p}$ is a $S$-ring. Let $\alpha=\sum x_{p}$ and $\beta=\Sigma y_{p}$ be elements in $R$ where $x_{p}, y_{p} \in R_{p}\left(x_{p}=y_{p}=0\right.$ for almost all $p$ 's). Since, for each $p, x_{p} y_{p}=a_{p} x_{p}+b_{p} y_{p}$ for constants $a_{p}$ and $b_{p}$, $\alpha \beta=\sum x_{p} y_{p}=\Sigma a_{p} x_{p}+\sum b_{p} y_{p}$. For each $p$, let $n_{p}=\max \quad\left(o\left(x_{p}\right)\right.$, $\left.o\left(y_{p}\right)\right)$. By the Chinese Remainder Theorem, there exist constants $k$ and 1 satisfying $k \equiv a_{p}\left(\bmod n_{p}\right)$ and $1 \equiv b_{p}\left(\bmod n_{p}\right)$ for each $p$ for which $x_{p}$ or $y_{p}$ is nonzero. It follows that $\alpha \beta=k \alpha+1 \beta$ and that $R$ is a $S$-ring.

2. Torsion-free rings. In this section all rings are assumed to be torsion-free. We first establish, in 5 lemmas, some properties of $S$-rings. Theorem 2.6 will be the culmination of this effort.

LEMMA 2.1. If $x$ and $y$ are elements in a $S$-ring, $x^{2}=r x$, and $y^{2}=s y$, then $x y+y x=s x+r y$ and $x y=s x$ or $r y$.

Proof. First, suppose $x$ and $y$ are linearly independent. The proof that $x y+y x=s x+r y$ is the same as in (1.2), except that $k$ now equals $s+r$ exactly. Next, set $x y=a x+b y, y x=c x+d y$. Then, $a r x+b r y=$ $x^{2} y=x(x y)=(a r+a b) x+b^{2} y$ and $a b=0=b(b-r)$. Similarly, $c d=$ $0=c(c-r)$. If $b \neq 0$, then $b=r, a=0$, and $x y=r y$. If $c \neq 0$, then $c=r, d=0$, and $y x=r x$ which means that $x y=s x$. If $b=c=0$, then $x y=s x$ since $a+c=s$. Secondly, suppose $x$ and $y$ are linearly dependent and $m x=n y \neq 0$. By computation, $m x y=m(s x)$ and $x y=$ $s x$. Similarly, $y x=r y$, and, as a result, $x y+y x=s x+r y$. 
Lemma 2.2. For elements $x$ and $y$ in $a$ S-ring the following are equivalent:

(1) $x^{2}=y^{2}=0$

(2) $x y=y x=0$,

(3) $x y+y x=0$.

Proof. From (2.1), (1) implies (2) and (2) implies (3). We must show that (3) implies (1). If $x$ and $y$ are linearly independent, this follows from (2.1). Suppose $m x=n y \neq 0$. Then $0=m n(x y+y x)=$ $m(x(n y)+(n y) x)=m(x(m x)+(m x) x)=2 m^{2} x^{2}$ and $x^{2}=0$. Similarly, $y^{2}=0$.

Lemma 2.3. If $K$ is a subring of the $S$-ring $R$ and $K^{2}=0$, then $K$ is an ideal.

Proof. Let $x \in K, y \in R \backslash K$. If $x$ and $y$ are linearly dependent, then $x y=y x=0 \in K$. Assume that they are linearly independent and write $x y=a x+b y$. Then $0=x^{2} y=x(x y)=x(a x+b y)=b x y=$ $a b x+b^{2} y$ and $b y=0$. Thus, $x y$ (similarly $y x$ ) is in $K$.

LEMMA 2.4. If the $S$-ring $R$ has rank one, it is a zero-ring or $R^{+}$is cyclic.

Proof. Assume the lemma to be false. Then there is an element $x \neq 0$ in $R^{+}$of unbounded height. Since $R^{2} \neq 0, x^{2}=a x \neq 0$ for some $a$. Choose $n$ and $y$ such that $n y=x$ but $n$ is not a factor of $a$. If $y^{2}=b y$, by computation $a x=n^{2} b y=n b x$ and $n$ divides $a$, a contradiction. Therefore, the lemma is true.

LEMMA 2.5. If $R$ is a $S$-ring, then it is a zero-ring or it contains an ideal $K$ and an element $z$ such that $R^{+}=K^{+} \oplus\langle z\rangle$, where $K^{2}=0$ and $z^{2}=s z$ for some $s \neq 0 . \quad$ Also, for every $x$ in $K, x z=u x$ and $z x=(s-u) x$ where $u(s-u)=0$.

Proof. Suppose $R$ is not a zero-ring and $K$ is a maximal subring in $R$ such that $K^{2}=0$. By (2.3), $K$ is an ideal and we may form the quotient ring $R / K=L$. We will show that $L^{+}$is cyclic and torsionfree. First, $L$ has property $S$. For, if $D$ is a subgroup of $L^{+}$, so is its inverse image in $R^{+}$and closure under multiplication in $R$ implies it in $L$. Secondly, $L$ is torsion-free. Suppose $y \neq 0$ but $n y=0$ in $L$. If $x$ in $R$ maps to $y$, then $n x$ is in $K$ and $(n x)^{2}=0$. If $x^{2}=0$, the subring generated by $x$ and $K$ is a zero-ring by (2.2) which contradicts the maximality of $K$. Thus, $x^{2} \neq 0, n=0$, and $L$ is torsion-free. Thirdly, we prove that $L$ has rank one. By the previous argument, it suffices to 
show that $r(L)>1$ implies $R \backslash K$ has a nonzero element whose square is zero. Suppose, then, $r(L)>1$ and $x$ and $y$ are linearly independent in $R \backslash K$. If $x^{2}=r x, y^{2}=s y$, then, by $(2.1), \quad x y+y x=s x+r y$ and $(s x-r y)^{2}=0$. Therefore, the rank of $L$ must be one. By the maximality of $K, L$ is not a zero-ring. By (2.4), $L^{+}$is cyclic and $R^{+}=K^{+} \oplus\langle z\rangle$ for $z$ where $z^{2} \neq 0$. Finally, we verify the last sentence of the theorem. Let $x, y$ be elements in $K$. By (2.1) it will suffice to show that $y z=u y$ implies $x z=u x$. Since $K$ is an ideal, $x z=a x$ for some a. Suppose $x$ and $y$ are linearly independent. Then $(x+y) z=$ $a x+u y$ must be a multiple of $x+y$ and $x z=u x$. Suppose $m x=$ $n y \neq 0$. Then $\max =m x z=n y z=n u y=u m x \quad$ and $\quad a=u, \quad$ as desired. The proof is complete.

We can now describe all $S$-rings on torsion-free groups.

THEOREM 2.6. Let $G$ be a torsion-free group of the form $H \oplus\langle z\rangle$. A $S$-ring can be constructed on $G$ by, for $x, y \in H$, setting $(x+a z)(y+b z)=b u x+a(s-u) y+a b s z$, where $u(s-u)=0$. Any torsion-free $S$-ring, not a zero-ring, has such a structure.

Proof. If $R$ is a $S$-ring and $R^{2} \neq 0$, then the structure of $R$ is as above by (2.5). Conversely, suppose the above structure is given. It is easy to check that multiplication is well-defined and that the distributive law holds. Let $\alpha=x+a z, \beta=y+b z, \gamma=w+c z$, where $x, y$, $w \in H$. We may assume $u=0$ (the proof is similar if $u=s$ ). Then $\alpha \beta=a s \beta$ and property $S$ is assured. Since $(\alpha \beta) \gamma=($ as $)(b s) \gamma=$ $\alpha(\beta \gamma)$, the associative law is satisfied. Therefore, the construction defines a $S$-ring.

3. Mixed rings. In this section the ring $R$ is always mixed and $T$ is its torsion subring. It is well-known that the torsion subring of a ring is an ideal. If $R$ is a $S$-ring, we can say more.

Proposition 3.1. If $R$ is a $S$-ring, $T^{2}=0$.

Proof. We first show that $x^{2}=0$ for every $x$ in $T$. Let $x \in T$, $y \in R \backslash T$, where $o(x)=n, \quad x^{2}=r x$, and $y^{2}=s y$. Then $(x+n y)^{2}=$ $r x+n^{2} s y=k(x+n y)$ for some $k$. Hence, $k=n s$ and $x^{2}=n s x=$ 0 . To complete the proof, it will suffice to show $x z=0$ for linearly independent $x$ and $z$ in $T$. We write $x z=a x+b z$ and, since $T$ is an ideal, $y z=c z$. Then $(x+y) z=a x+(b+c) z$ and $a x$ must be a multiple of $x+y$ which implies $a x=0$. Similarly, we can show $b z=0$ by considering $x(z+y)$. Therefore, $x z=0$ and, as a result, $T^{2}=0$. 
If $R$ is a mixed $S$-ring, not a zero-ring, then $R / T$ may or may not be a zero-ring. We consider the second case first and describe the structure of $R$ when $(R / T)^{2} \neq 0$ in Theorem 3.5. To prove it three lemmas are required.

LEMMA 3.2. If $R$ is a $S$-ring and $R / T$ is not a zero-ring, then $R$ has an ideal $K$ containing $T$ and an element $z$ such that $K^{2}=0, z^{2} \neq 0$, and $R^{+}=K \oplus\langle z\rangle$.

Proof. By (2.5), $(R / T)^{+}=(K / T) \oplus\langle z+T\rangle$ for some ideal $K / T$ and element $z+T$ such that $(K / T)^{2}=0,(z+T)^{2} \neq 0$. By the isomorphism theorems for groups, $R^{+}=K^{+} \oplus\langle z\rangle$, where $K \supset T$ and $z^{2} \neq 0$. Since $T$ and $K / T$ are ideals, so is $K$. We must show $K^{2}=0$. Since any two elements of $K$ are contained in a subgroup of torsion-free rank at most two, it will suffice to show $K^{2}=0$ where $K^{+}=T \oplus\langle x\rangle \oplus\langle y\rangle$ for some $x$ and $y$. Since $K / T$ is a zero-ring, so is $\langle x\rangle \oplus\langle y\rangle$ as well as $T$. We will show $t x=0$ for $t$ in $T$ and it will follow, by symmetry, that $K^{2}=$ 0 . Since $T$ and $K$ are ideals, $t x=a t, z x=b x$ for some $a$ and $b$. Then, $(t+z) x=a t+b x$ and $a t$ is a multiple of $t+z$ which implies $t x=a t=$ 0 . This completes the proof.

For the rest of this section, $T_{i}$ is the $p_{i}$-component of $T$ where $\left\{p_{i}\right\}$, $i \in I$, is the set of prime numbers.

Lemma 3.3. Let $R$ be a $S$-ring, $R^{+}=K \oplus\langle z\rangle$, where $K \supset T, K^{2}=$ 0 , and $z^{2}=s z$. Then:

(1) if $K$ is mixed, for every $x$ in $K, x z=u x, z x=(s-u) x$ where $u(s-u)=0$;

(2) if $K=\oplus T_{1}$, there exists $u_{\imath}$ for each $i$ such that $x_{1} z=u_{1} x_{t}$, $z x_{i}=\left(s-u_{i}\right) x_{i}$, and $u_{i}\left(s-u_{i}\right) x_{t}=0$ for every $x_{i}$ in $T_{\imath}$.

Before proving this lemma, we establish the following.

Lemma 3.4. Let $R$ be as in (3.3). If $x, y \in K$ and $o(y)$ is infinite or $o(x)$ divides $o(y)$, then $y z=u y$ implies $x z=u x$.

Proof. If $x$ and $y$ are linearly independent or if $o(x)$ is infinite, the argument at the end of the proof of (2.5) may be used to prove the lemma. If $x$ and $y$ are dependent and $o(x)$ is a finite number, then $x=w+a y$ for element $w$ and constant $a$ such that $w$ is independent of $y$ and $o(w)$ divides $o(y)$. By the previous sentence applied to $w$ and $y$, $w z=u w$. Again $x z=u x$.

Proof of 3.3. In general, if $x \in K$ and $x z=u x$, then $z x=(s-u) x$ and $u(s-u) x=0$. To see this, consider $(x+z)^{2}=x z+z x+s z$. Since 
this expression must be a multiple of $x+z, x z+z x=s x$ or $z x=$ $(s-u) x$. Also, $\quad s u x=s x z=x(s z)=x z^{2}=u^{2} x \quad$ and $\quad u(s-u) x=$ 0 . Suppose, then, $K$ is mixed. If $y \in K \backslash T$, and $y z=u y$, then $u(s-u)=0$ and, by (3.4), $x z=u x$, for every $x$ in $K$. We have proved (1). Assume, then, $K=T$ as in (2). We will examine the following possible cases: (a) $T_{i}$ is bounded, (b) $T_{1}$ is not reduced, and (c) $T_{1}$ is unbounded and reduced. (a) Let $T_{1}$ be bounded. Let $y$ be an element of maximal order in $T_{1}$ and $y z=u_{i} y$. Then (2) follows from (3.4) and from what was said at the beginning of this proof. (b) Let $T_{i}^{+}$contain a divisible subgroup $D$ of rank one. We claim $D z=0$ or $z D=$ 0 . Suppose this is not so. Then $D$ contains an element $y$ such that neither $y z$ nor $z y$ is zero. For $n>0$, there exists $x$ such that $p^{n} x=y$, and for some $u, x z=u x$ and $z x=(s-u) x$ by what was said above. But, if $n$ is large relative to $o(y), u y$ or $(s-u) y$ is zero since $u(s-u) x=0$, although $u y=y z \neq 0 \neq z y=(s-u) y$. From this contradiction we conclude that $D z=0$ or $z D=0$ and $y z=u y$ for all $y$ in $D$ where $u(s-u)=0$. If $x \in T_{\imath} \backslash D$, we may select $y \in D$ such that $o(x)$ divides $o(y)$ and (3.4) completes tne proof of (2) in case (b). (c) Suppose $T_{1}$ is reduced with unbounded basic subgroup $B$. First, assume $B z \neq 0 \neq z B$. We show a contradiction. If $x z \neq 0 \neq z y$ for $x, y$ in $B$ and $o(x)$ divides $o(y)$, then $y z \neq 0$ by (3.4). In this event, we select $w \in B$ of large order relative to that of $y$. Then, if $w z=u w, z w=$ $(s-u) w, \quad u y=y z \neq 0 \neq z y=(s-u) y$. But $u(s-u) w=0$ and the largeness of $o(w)$ imply $u y$ or $(s-u) y$ equals zero. Therefore, $B z=0$ or $z B=0$ and, for all $x \in B, x z=u x$ where $u(s-u)=0$. If $g \in T_{i} \backslash B$, we may choose $x \in B$ such that $o(g) \leqq o(x)$ and, by (3.4), gz=ug. We have established (2) for case (c).

THEOREM 3.5. Let $G=H \oplus\langle z\rangle$ be a mixed group where $H \supset$ $T$. A $S$-ring $R$ can be constructed on $G$ by defining:

(1) if $H \neq T,(x+a z)(y+b z)=a(s-u) y+b u x+a b s z$ for $x$, $y \in H$ where $u(s-u)=0$;

(2) if $\quad H=\oplus T_{t}, \quad\left(\sum x_{\imath}+a z\right)\left(\sum y_{t}+b z\right)=a \sum\left(s-u_{i}\right) y_{\imath}+b \sum u_{\imath} x_{i}$ + absz for $x_{i}, y_{1} \in T_{1}$ where $u_{i}\left(s-u_{i}\right) T_{i}=0$ for each $i$. If $R$ is a mixed $S$-ring and $R / T$ is not a zero-ring, $R$ has the above structure with $s \neq 0$.

Proof. We prove the last sentence first. If $R$ is a mixed $S$-ring and $R / T$ is not a zero-ring, $R$ has the structure of the theorem by (3.2), (3.3), and the distributive law. Therefore, we assume $G$ is as given and show that (1) and (2) define $S$-rings on $G$. The proof for definition (1) is the same as in (2.6). We assume (2) is given. A routine calculation shows that multiplication is well-defined and that the distributive law holds. This leaves associativity and property $S$ to be verified. Let 
$\alpha=\sum x_{t}+a z, \beta=\sum y_{i}+b z, \gamma=\sum w_{t}+c z$, where $x_{i}, y_{i}, w_{i} \in T_{i}$ and let $n_{i}=\max \left(o\left(x_{i}\right), o\left(y_{i}\right), o\left(w_{i}\right)\right)$. By the Chinese Remainder Theorem, we can find $k$ such that $k \equiv u_{i}\left(\bmod n_{t}\right)$ for each $i$ such that $x_{i}, y_{i}$, or $w_{i}$ is nonzero. Then $\alpha \beta=b k \alpha+a(s-k) \beta$ and property $S$ is assured. Finally, $(\alpha \beta) \gamma=b c k^{2} \alpha+a c k(s-k) \beta+a b s(s-k) \gamma=\alpha(\beta \gamma)$ and the ring is associative. The proof is complete.

If $R$ is a $S$-ring, it may happen that $R / T$ is a zero-ring but $R$ is not. In this case, as we shall see, the additive group of $R / T$ has rank one but may not be cyclic. Hence, a few words on mixed groups of torsion-free rank one are in order. Let $G$ be such a group and $T=\bigoplus T_{v}, i \in I$, its torsion subgroup ( $T_{i}$ is the $p_{i}$-component of $T$ where $p_{\imath}$ is a prime number). Let $z$ be a torsion-free element in $G$. We partition $I$ into subsets $J$ and $K$ such that $i$ is in $J$ or $K$ exactly if the $p_{i}$-height of $z+T$ in $G / T$ is infinite or finite, respectively. The latter height is designated $n_{v}$. In this case $G$ has a generating set:

$$
A=\left\{T_{i}, z_{j n}, z_{k}\right\}, i \in I, j \in J, k \in K, \quad n=1,2, \cdots
$$

where

$$
\begin{aligned}
z & =p_{l}^{n} z_{j n}+t_{j n}, \quad t_{j n} \in T \\
& =p_{k}^{n_{k}} z_{k}+t_{k}, \quad t_{k} \in T_{k} .
\end{aligned}
$$

Here $z_{k}=z$ if $n_{k}=0$. We write $G=(A, *)$. Each element $\alpha$ in $G$ has a unique expression of the form:

$$
\alpha=\sum x_{\imath}+\sum_{\jmath} a_{j} z_{\jmath m,}+\sum b_{k} z_{k}+c z
$$

where $x_{l} \in T_{l}, m_{l}$ depends on $j,\left(a_{l}, p_{j}\right)=1,\left|a_{l}\right|<p_{j}^{m_{j}}$, and $\left|b_{k}\right|<p_{k}^{n_{k}}$. The last sentence can be seen by examining the image of $\alpha$ in $G / T$.

We are ready to state and prove the final theorem of this section.

THEOREM 3.6. Let $G=(A, *)$. A $S$-ring $R$ can be constructed on $G$ in the following manner. For each $i$ in $I$, choose $u_{\imath}$ such that $u_{\imath}^{2} T_{i}=0$ and, if $i \in J, u_{i}=0$. For each $i$ and $k \neq i \neq j$, choose $u_{k i}$ and $u_{j i}$ such that $u_{k l}=0=u_{j i}$ if $T_{i}$ is unbounded and $p_{k} u_{k i} \equiv 1 \equiv p_{1} u_{j i}\left(\bmod p_{i}^{N_{i}}\right)$ if $T_{1}$ has least bound $p_{\imath}^{N_{i}}$. Multiplication on $G$ is defined by the following rules:

(1) $T^{2}=0$, and $x y=-y x$ for $x, y \in A$.

(2) For $x_{i} \in T_{l}, z_{k}$, and $z_{j n}$,

$$
x_{i} z_{k}=u_{k i}^{n_{k}} p_{i}^{n_{i}} u_{i} x_{i}
$$


and

$$
x_{i} z_{j n}=u_{j i p}^{n} p_{i}^{n_{1}} u_{i} x_{i} \quad\left(n_{t}=0 \quad \text { if } \quad i \in J\right)
$$

(3) For $i \in K$ and $z_{k}, z_{\jmath n}$,

$$
z_{1} z_{k}=u_{i k}^{n_{i}} u_{k} t_{k}-u_{k i}^{n_{k}} u_{i} t_{i}
$$

and

$$
z_{\imath} z_{j n}=-u_{j i}^{n} u_{t} t_{v}
$$

(4) For $i \in J$ and $z_{\text {เm }}, z_{\text {jn }}$,

$$
z_{\text {ım }} z_{j n}=0
$$

(5) Multiplication on $G$ is linear relative to $A$. If $R$ is a $S$-ring and $R / T$ but not $R$ is a zero-ring, then $R$ has the above structure.

Proof. (a) We prove the last sentence first. Assume $S$-ring $R$ is not a zero-ring but $R / T$ is. We claim $r(R / T)=1$. To show this, it will suffice to prove $R$ is a zero-ring if $R^{+}=T \oplus\langle x\rangle \oplus\langle y\rangle$, for some $x$ and $y$. We know $T^{2}=0$. Since $R / T$ is a zero-ring, so is the ring on $\langle x\rangle \oplus\langle y\rangle$. If $t \in T,(t+y) x=t x$ is in $T$ and must be a multiple of $t+y$. Thus, $t x=0$. Other products in $R$ are zero by symmetry, and $R^{2}=0$. Therefore, $r(R / T)=1$, and we let $R^{+}=G=(A, *)$. We proceed to verify properties $1-5$. Throughout this proof we assume $k$ and $j$ are always in $K$ and $J$ respectively. First, since $T$ and $R / T$ are zero-rings, it follows that, for $x, y \in R, x^{2}=y^{2}=0=(x+y)^{2}=x y+y x$ and, hence, (1) is necessary. If $i \in K$, consider the subgroup $T_{1} \oplus\left\langle z_{1}\right\rangle$. By (3.3) with $s=0$, there is a constant $u_{1}$ such that $u_{1}^{2} T_{1}=0$ and $x_{i} z_{t}=u_{1} x_{t}$, for every $x_{t}$ in $T_{1}$. If $i \in J$ and $x_{t} \in T_{l}, x_{t} z_{t n}=0$ for all $n$ because of the height condition on $z_{\imath n}$ (modulo $T_{1}$ ) and, as a result, $x_{i} z_{\text {in }}=u_{i} x_{i}$ where $u_{i}=0$. We have proved (2) for $i=k$ or $j$. Next, for fixed $i$ and $k \neq i \neq j$, let $u_{k i}$ and $u_{j i}$ be as stated in the theorem. If $i \in K$, by reason of $(*)$,

$$
p_{i}^{n_{i}} u_{i} x_{i}=x_{i}\left(p_{i}^{n_{i}} z_{i}+t_{i}\right)=x_{i}\left(p_{k}^{n_{k}} z_{k}+t_{k}\right)=x_{i}\left(p_{j}^{n} z_{j n}+t_{j n}\right) .
$$

If $i \in J$, the same equations hold true when, for $m>0, p^{m}$ and $z_{l m}$ replace $p_{\imath}^{n_{t}}$ and $z_{\imath}$, respectively. These equations, then, imply (2) for the remaining possible cases. We next verify (3). By (1), any element in $A$ squared is zero and (3) is true for $i=k$. For $i \in K$ but $i \neq k$, consider $p_{i}^{n_{i}} z_{i}+t_{i}=p_{k}^{n_{k}} z_{k}+t_{k}$. Multiplying on the right (left) by $z_{k}\left(z_{i}\right)$, we derive: 


$$
p_{i}^{n_{i}} z_{l} z_{k}=u_{k} t_{k}-u_{k i}^{n_{k}} p_{l}^{n_{i}} u_{i} t_{l} \quad \text { and } \quad p_{k}^{n_{k}} z_{l} z_{k}=u_{i k}^{n_{k}} p_{k}^{n_{k}} u_{k} t_{k}-u_{l} t_{l}
$$

Since $z_{\imath} z_{k} \in T_{k} \oplus T_{\imath}, z_{\imath} z_{k}$ has the form given in (3). Similarly, considering $p_{i}^{n_{i}} z_{i}+t_{i}=p_{l}^{n} z_{j n}+t_{j n}$ for $i \in K$ and multiplying right (left) by $z_{j n}\left(z_{\imath}\right)$, we derive the second equation of (3). We next prove (4). For $i \in J$ but $i \neq j$, consider $p^{m}{ }_{1} z_{l m}+t_{t m}=p^{n} z_{j n}+t_{j n}$. If we multiply right (left) by $z_{j n}$ $\left(z_{l m}\right)$, we obtain $p_{i}^{m} z_{l m} z_{j n}=0=p_{j}^{n} z_{l m} z_{j n}$ which implies $z_{l m} z_{j n}=$ 0 . Suppose, however, $i=j$ and $m>n$. Then $z_{j n}=p^{m-n} z_{\imath m}+x_{\imath}$ for some $x_{i} \in T_{l}$, and $z_{l m} z_{l n}=p^{m-n} z_{\imath m}^{2}+z_{l m} x_{\imath}=0$. We have proved (4). Since (5) is obvious, the proof of the final sentence of the theorem is complete. (b) Suppose $G$ and multiplication on $G$ are as given in the theorem. We must prove that multiplication is well-defined and that the associative and $S$ properties are present. We first observe that the second equation of (1) is consistent with the first equation of (3) and of (4). We now prove multiplication is well-defined. First, if $x \in A$ and $y, y_{1}$ are two representatives of $z$ given by $(*)$, we claim $x y=x y_{1}$ and it is meaningful to write $x z=x y=x y_{1}$. To prove this, we consider cases. If $x \in T_{1}$, a reversal of the proof of (2) above yields the desired result. If $x=z_{i}, i \in K$, a reversal of the proof of (3) above yields the desired result. If $x=z_{j n}$, then $x y=0$ for every representative $y$ of $z$. We compute the less obvious product. Let $z=p_{i}^{n_{i}} z_{i}+t_{t}$, $i \in K$. Then

$$
\begin{aligned}
& z_{j n}\left(p_{i}^{n_{i}} z_{i}+t_{i}\right)=-p_{i}^{n_{i}} z_{i} z_{j n}-t_{i} z_{j n} \\
& =p_{\imath}^{n_{i}} u_{l}^{n}{ }_{l}^{n} u_{l} t_{l}-u_{j i}^{n} p_{i}^{n_{i}} u_{l} t_{l}=0 .
\end{aligned}
$$

Secondly, if $x \in A$ and $z_{j n}=p_{\jmath} z_{j n+1}+x_{j n}$ where $x_{j n} \in T_{j}$, we claim $x z_{j n}=$ $x\left(p_{j} z_{j n+1}+x_{j n}\right)$. Again we consider cases. If $x \in T_{\imath}$, (2) yields the desired result. If $x=z_{\imath}, i \in K$, then, by (3),

$$
\begin{aligned}
z_{\imath} z_{j n} & =-u_{j i}^{n} u_{\imath} t_{\imath}=p_{J}\left(-u_{j i}^{n+1} u_{i} t_{\imath}\right)=p_{\jmath}\left(z_{i} z_{j n+1}\right) \\
& =z_{\imath}\left(p_{\jmath} z_{j n+1}+x_{j n}\right)
\end{aligned}
$$

as desired. If $x=z_{\imath m}, \quad i \in J$, then $z_{\imath m} z_{j n}=0=z_{\imath m}\left(p_{\jmath} z_{j n+1}+x_{j n}\right)$, as desired. Thirdly, multiplication on all of $G$ is well-defined. By reason of (1) and (5) it will suffice to show that $\alpha \beta=\alpha^{\prime} \beta$ where $\alpha=\alpha^{\prime}, \alpha$ is the representative of $(* *)$, and $\beta$ is arbitrary in $G$. Suppose, then, $\alpha^{\prime}=$ $\sum_{i} y_{i}+\sum_{j}\left(\sum_{n} d_{j n} z_{j n}\right)+\sum_{k} e_{k} z_{k}+f z$ where $y_{1} \in T_{l}$. For fixed $j$ and $n<m_{j}$, by what was said above, $d_{j n} z_{j n}$ may be replaced by $d_{j n} p_{j} z_{j n+1}+d_{j n} x_{j n}$ in computing $\alpha^{\prime} \beta$. If $n>m_{\text {, }}$, by the uniqueness of the form of $\alpha, d_{j n}=p_{j} d_{j n}^{\prime}$ for some $d_{j n}^{\prime}$ and $d_{j n} z_{j n}=d_{j n}^{\prime} p_{j} z_{j n}=d_{j n}\left(z_{j n-1}-x_{j n-1}\right)$. We replace the left term by the right term in computing $\alpha^{\prime} \beta$. Continuing in this manner and simplifying, for each $j$, we may replace $y_{j}+\Sigma_{n} d_{j n} z_{j n}$ by $x_{\jmath}+a_{l} z_{j m_{j}}+f_{j} z$ for 
some $f_{j}$. In like manner, for each $k$, we may replace $y_{k}+e_{k} z$ by $x_{k}+b_{k} z_{k}+f_{k} z$ for some $f_{k}$. The sum of the $f_{j}$ 's, $f$, and the $f_{k}$ 's must equal $c$ and it follows that $\alpha^{\prime} \beta=\alpha \beta$. Multiplication is well-defined. Finally, we establish the associative law and property $S$. It will suffice to show them for a subring $R_{1}$ of $R$ where $R_{1}$ is finitely generated over $T$. Since any finitely generated subgroup of the group $G / T$ is cyclic, we may assume $R_{1}^{+}=T \bigoplus\langle y\rangle$ where $y$ is a linear combination of members of $A \backslash T$. By reason of (1) and (2) and linearity, there exists a constant $v_{\imath}$ for each $i$ such that $x_{i} y=v_{i} x_{i}=-y x_{i}$ for every $x_{i}$ in $T_{i}$ and $v_{i}^{2} T_{t}=0$. By the proof of (3.5), $R_{1}$ is associative and possesses property $S$. The proof of the theorem is complete.

4. Special properties. The additive group of an Artinian, Noetherian, or regular ring belongs to a narrowly defined and wellknown class of groups (see chapter XVII of [4]). This knowledge together with the theorems of the preceding sections enable us to decide the nature of particular classes of $S$-rings. We describe the classes that seem most important in the following corollaries.

Corollary 4.1. A $S$-ring $R$ has an identity if and only if $R$ is isomorphic to $Z$, to $Z /(2) \oplus Z /(2)$ (ring-direct sum ), or to $\oplus Z /\left(p^{n_{p}}\right)$ summed over a finite number of distinct primes $p$ where $n_{p}$ depends on $p$.

COROLLARY 4.2. The rings $Z$ and $Z /(p)$ are the only integral domains with property $S$.

COROLlaRY 4.3. A nontrivial $S$-ring $R$ is regular if and only if it has the form $\oplus Z /(p)$ (ring-direct sum) where each $p \neq 2(p=2)$ occurs at most once (twice).

COROllary 4.4. A $S$-ring $R$ is Artinian if and only if it is a torsion ring of finite rank.

COROllary 4.5. A $S$-ring $R$ is Noetherian if and only if $R^{+}$is a direct sum of a finite number of cyclic groups.

In the following let $T$ be the torsion subring of $R$ and $T_{i}$ be the $p_{i}$-component of $T$ for prime number $p_{i}$. Let $\oplus$ signify ring-direct sum and let $(z)$ and $\left(z_{\imath}\right)$ signify the subring generated by $z$ and $z_{\imath}$, respectively.

COROLlary 4.6. A ring $R$ has the property that every additive subgroup is an ideal if and only if

(1) $R$ is a zero-ring,

(2) $\quad R=T \oplus(z)$ and $T^{2}=s T$ where $z^{2}=s z$, or 
(3) $\quad R=\bigoplus T_{i}$ and, for $T_{i}^{2} \neq 0, T_{i}=H_{i} \oplus\left(z_{i}\right)$ and $H_{i}^{2}=0=s_{i} H_{i}$ where $z_{i}^{2}=s_{i} z_{i}$.

The last corollary is not new and may be found in [5]. We include it here because it follows readily from the theorems of the paper.

\section{REFERENCES}

1. R. A. Beaumont, Rings with additive group which is the direct sum of cyclic groups, Duke Math. J., 15 (1948), 367-369.

2. R. A. Beaumont and R. S. Pierce, Torsion-free rings, Illinois J. Math., 5 (1961), 61-98.

3. L. Fuchs, Abelian Groups, Publ. House Hungar. Acad. Science, Budapest, 1958.

4. L. Fuchs, Infinite Abelian Groups, Academic Press, N. Y., Vol. I (1970), Vol. II (1973).

5. L. Redei, Die Vollidealringe, Monatshefte für Mathematik, 56 (1952), 89-95.

Received February 23, 1976.

UNIVERSITY OF DETROIT 


\section{PACIFIC JOURNAL OF MATHEMATICS}

\section{EDITORS}

RICHARI) ARENS (Managing Editor)

University of California

Los Angeles, CA 90024

R. A. Beaumiont

University of Washington

Seattle, WA 98105

\section{J. DugunduI}

Department of Mathematics University of Southern California Los Angeles, CA 90007

D. Gilbarg and J. Milgram Stanford University

Stanford, CA 94305

\section{ASSOCIATE EDITORS}

E. F. BECKENBACH
B. H. NEUMANN

F. Wolf

K. YoshidA

\section{SUPPORTING INSTITUTIONS}

UNIVERSITY OF BRITISH COLUMBIA CALIFORNIA INSTITUTE OF TECHNOLOGY

UNIVERSITY OF CALIFORNIA

MONTANA STATE UNIVERSITY

UNIVERSITY OF NEVADA

NEW MEXICO STATE UNIVERSITY

OREGON STATE UNIVERSITY

UNIVERSITY OF OREGON

OSAKA UNIVERSITY

\author{
UNIVERSITY OF SOUTHERN CALIFORNIA \\ STANFORD UNIVERSITY \\ UNIVERSITY OF HAWAII \\ UNIVERSITY OF TOKYO \\ UNIVERSITY OF UTAH \\ WASHINGTON STATE UNIVERSITY \\ UNIVERSITY OF WASHINGTON \\ AMERICAN MATHEMATICAL SOCIETY
}

The Supporting Institutions listed above contribute to the cost of publication of this Journal, but they are not owners or publishers and have no responsibility for its contents or policies.

Mathematical papers intended for publication in the Pacific Journal of Mathematics should be in typed form or offset-reproduced (not dittoed), double spaced with large margins. Underline Greek letters in red, German in green, and script in blue. The first $p: 1<$ graph or two must be capable of being used separately as a synopsis of the entire paper. Items of the biblography should not be cited there unless absolutely necessary, in which case they must he identified by author and Journal, rather than by item number. Manuscripts, in duplicate, may be sent to any one of the four editors. Please classify according to the scheme of Math. Reviews, Index to Vol. 39. All other communications should be addressed to the managing editor, or Elaine Barth, University of California, Los Angeles, California, 90024.

100 reprints are provided free for each article, only if page charges have been substantially paid. Additional copies may be obtained at cost in multiples of 50 .

The Pacific Journal of Mathematics is issued monthly as of January 1966. Regular subscription rate: $\$ 72.00$ a year (6 Vols., 12 issues). Special rate: $\$ 36.00$ a year to individual members of supporting institutions.

Subscriptions, orders for back numbers, and changes of address should be sent to Pacific Journal of Mathematics, 103 Highland Boulevard, Berkeley, California, 94708.

PUBLISHED BY PACIFIC JOURNAL OF MATHEMATICS, A NON-PROFIT CORPORATION Printed at Jerusalem Academic Press, POB 2390, Jerusalem, Israel.

\section{Copyright (C) 1976 Pacific Journal of Mathematics} All Rights Reserved 


\section{Pacific Journal of Mathematics}

\section{Vol. 66, No. 2 December, 1976}

Gerald A. Beer, Tax structures whose progressivity is inflation neutral..... 305

William M. Cornette, A generalization of the unit interval............. 313

David E. Evans, Unbounded completely positive linear maps on

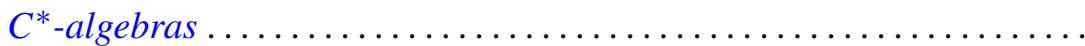

Hector O. Fattorini, Some remarks on convolution equations for

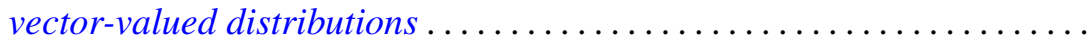

Amassa Courtney Fauntleroy, Automorphism groups of unipotent groups of

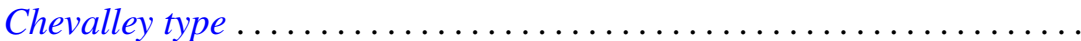

Christian C. Fenske and Heinz-Otto Peitgen, On fixed points of zero index in

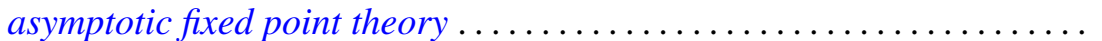

Atsushi Inoue, On a class of unbounded operator algebras. II ............

Herbert Meyer Kamowitz, The spectra of endomorphisms of algebras of

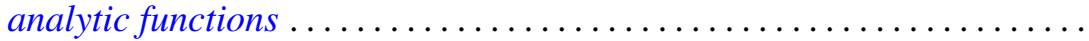

Jimmie Don Lawson, Embeddings of compact convex sets and locally compact cones ....................................

William Lindgren and Peter Joseph Nyikos, Spaces with bases satisfying certain order and intersection properties .....................

Emily Mann Peck, Lattice projections on continuous function spaces ...... 477

Morris Marden and Peter A. McCoy, Level sets of polynomials in $n$ real variables...

Francis Joseph Narcowich, An imbedding theorem for indeterminate Hermitian moment sequences......................

John Dacey O'Neill, Rings whose additive subgroups are subrings ...

Chull Park and David Lee Skoug, Wiener integrals over the sets bounded by sectionally continuous barriers .....................

Vladimir Scheffer, Partial regularity of solutions to the Navier-Stokes equations.

Eugene Spiegel and Allan Trojan, On semi-simple group algebras. II

Katsuo Takano, On Cameron and Storvick's operator valued function space integral 